\title{
Western Blotting Electrophoretic Sequencing: First Images with a Room Temperature CMOS Detector
}

\author{
M. Esposito ${ }^{1}$, J. Newcombe ${ }^{2}$ and K. Wells ${ }^{1}$
}

\begin{abstract}
CMOS imaging technology can be applied to Chemiluminescence Western Blotting as a potential imaging alternative technology to using conventional film-emulsion. In this work the authors present a through investigation on the performance of CMOS Active Pixel Sensors for using in western blotting. Chemiluminescence labeling is a well established technique to detect proteins and presents several advantages compared with the fluorescence labeling. In fact it requires neither external illumination nor filtering optics and does not produce an inherently label-related background to correct. In this paper the first results of imaging a secondary antibody labeled with chemiluminescence reagents obtained with a CMOS sensor operating at room temperature are presented.
\end{abstract}

Index Terms-CMOS, APS, Room temperature image sensors, Electrophoresis, Western blotting, Enhanced chemiluminescence.

\section{INTRODUCTION}

W ESTERN blotting electrophoretic sequencing is an analytical technique widely used in life sciences to detect, recognize and quantify specific labeled proteins in biological samples. A widely used label for western blotting are Enhanced ChemiLuminescence (ECL) reagents [1] based on fluorescent light emission at $425 \mathrm{~nm}$ of luminol (3-amino-phthalhydrazide) cathalized by oxidizing enzymes, such as horseradish peroxidase. Chemiluminescence labeling in western blotting electrophoresis and, more generally, in electrophoresis for genomics and proteomics represents a well established technique. In fact chemiluminescence labeling requires neither external illumination nor filtering optics and does not produce an inherently label-related background to correct. Film emulsion is the most common detecting medium for detecting these labels used in a variety of applications such as western blotting imaging. Nevertheless it presents key drawbacks such as a limited and non linear dynamic range. Furthermore chemiluminescence detection with film emulsion often requires multiple exposures to capture the full dynamic range of the optical emission: an expensive time consuming procedure. Conventional digital imaging techniques have been shown suitable as quantitative imaging detectors for chemiluminescence light, especially with the use of cooled Charged

This work was supported by the RC-UK Multidimensional Integrated Intelligent Imaging Plus (MI-3 Plus) programme (EP/G037671/1).

${ }^{1}$ Centre for Vision, Speech and Signal Processing, Faculty of Engineering and Physical Sciences, University of Surrey, Guildford GU2 7XH,U.K. (email:m.esposito@surrey.ac.uk ).

${ }^{2}$ Faculty of Health and Medical Sciences, University of Surrey, Guildford GU2 7XH,U.K.
Coupled Device (CCD) both as prototypes [2][3] and commercially available systems [4][5]. Even so these system present several limitations in imaging performance, such as low frame rate due to the sequential read out, and require operations at low temperature to achieve reasonable noise level. Other chemiluminescence systems based on integrated amorphous silicon thin-film photosensors [6] and single photon avalanche diode [7] have been tested in the last few years.

In this paper the authors present a novel ECL detection system based on a room temperature CMOS detector. The CMOS detector used here is a state-of-the-art CMOS detector, named Vanilla, specially designed for biomedical imaging applications by the MI3 consortium [8]. This sensor was successfully tested as a viable solution to use in autoradiography [9]. Results on the performance in ECL detection at room temperature and comparison with film images are reported.

\section{Materials And Methods}

Protein detection in western blotting is a multi-step process summarized in fig. 1. In fact in order to be detected, proteins need to be chemically bound in several stages until the last stage where light is emitted for detection. Proteins (antigens) bind a specific primary antibody, which in turn binds a secondary specific antibody. Secondary antibody are bound to $\mathrm{H}_{2} \mathrm{O}_{2}$ molecules in order to emit light when exposed to a Lumilol substrate, according to the following chemical reaction:

$$
\text { Luminol }+\left[\mathrm{H}_{2} \mathrm{O}_{2}\right] \rightarrow 3-A P A[\circ] \rightarrow 3-A P A+\gamma
$$

A $425 \mathrm{~nm}$ photon is emitted at the end of this reaction. In order to assess the response of the CMOS system under investigation to the chemiluminescent light produced by reaction of secondary antibody with luminol, only the last stage of the detection chain will be studied in this paper. The Vanilla active pixel sensor (APS) (fig. 2) consists of a $512 \times 512$ array of pixels on a $25 \mu \mathrm{m}$ pitch for an epitaxial thickness of $20 \mu \mathrm{m}$ and an active area of $13 \times 13 \mathrm{~mm}^{2}$. The readout is column-parallel with a 12-bit ADC per four columns driven by a specifically designed Field Programmable Gate Array (FPGA). Chemiluminescent samples were prepared using ECL labeled secondary antibody. A $70 \mu \mathrm{l}$ drop of antimouse IgG HorseRadish Peroxidase (HRP) antibody (A8924, Sigma-Aldrich) $0.6 \mathrm{mg} / \mathrm{ml}$ concentration was diluted (1/5000) in a Tris Buffered saline solution. The resulting solution was spotted on a Hybond - LEP membrane (Amersham 


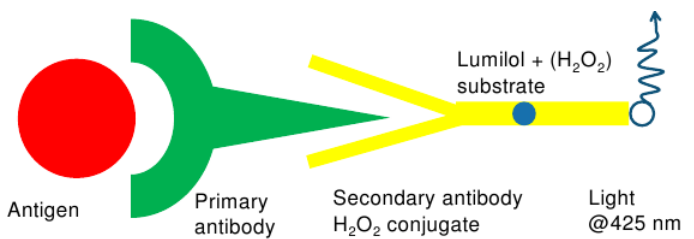

Fig. 1. A schematic representation of protein detection in western blotting. Proteins (antigens) bound a specific primary antibody, which in turns bind a specific secondary antibody. Secondary antibody are conjugated with $\mathrm{H}_{2} \mathrm{O}_{2}$ in order to emit light when exposed to a Luminol substrate.

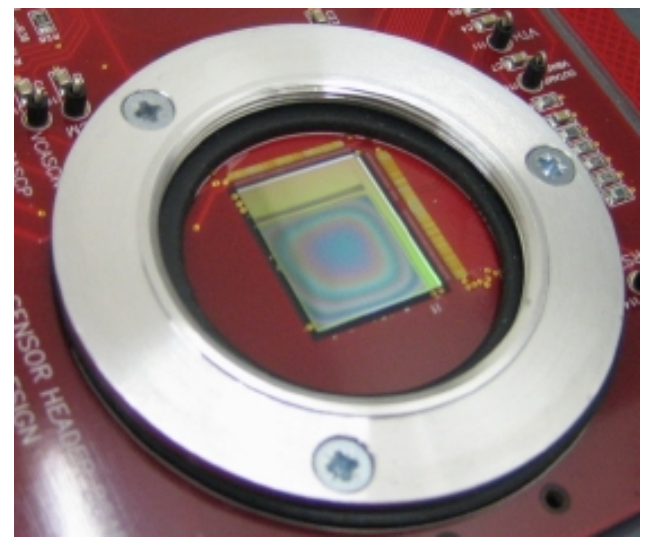

Fig. 2. A picture of the Vanilla CMOS image sensor used in this work.

Biosciences) The membrane was then washed in a LumiLight Western Blotting Substrate (Roche Applied Science) in order to activated the ECL emission by means of the Luminol contained. A second sample was prepared using antihuman IgG HRP antibody (A8667, Sigma-Aldrich) ranging from $117 \times 10^{-5}$ to $41 \times 10^{-2} \mu \mathrm{g}$. The resulting solutions were spotted on a Hybond - LEP membrane (Amersham Biosciences) The membranes were then washed in a LumiLight Western Blotting Substrate (Roche Applied Science) in order to activated the ECL emission. The experiment was set up placing the sensor in a light proof box and placing the sample in direct contact with the surface of the sensor for 5 hours, with images acquired at a frame rate of 1 frame per second. All the acquired data have been thresholded with respect to the per-pixel threshold $T_{i, j}$ :

$$
T_{i, j}=\mu_{i, j}^{r e f}+k\left[\sigma_{i, j}^{r e f}+\left(M^{r e f}-M^{c u r r}\right)\right]
$$

where $\mu_{i, j}^{r e f}$ is the mean value of the pixel $i, j$ in a reference dark acquisition, $\sigma_{i, j}^{r e f}$ is the standard deviation of the pixel $i, j$ in a reference dark acquisition, $M^{\text {curr }}$ is the global mode of the current frame and $M^{r e f}$ is the global mode of a reference dark acquisition. The term $\left(M^{\text {ref }}-M^{\text {curr }}\right)$ takes into account drifts in $\mu_{i, j}$ due to the thermal variation of the dark current and the constant $k$ was chosen equal to 2 in order to ensure a confidence interval of $95 \%$.

\section{RESULTS}

\section{A. Decay curve}

A $70 \mu \mathrm{l}$ drop of anti-mouse HRP antibody was imaged for 8 hours at 1 frame per second. The signal generated by

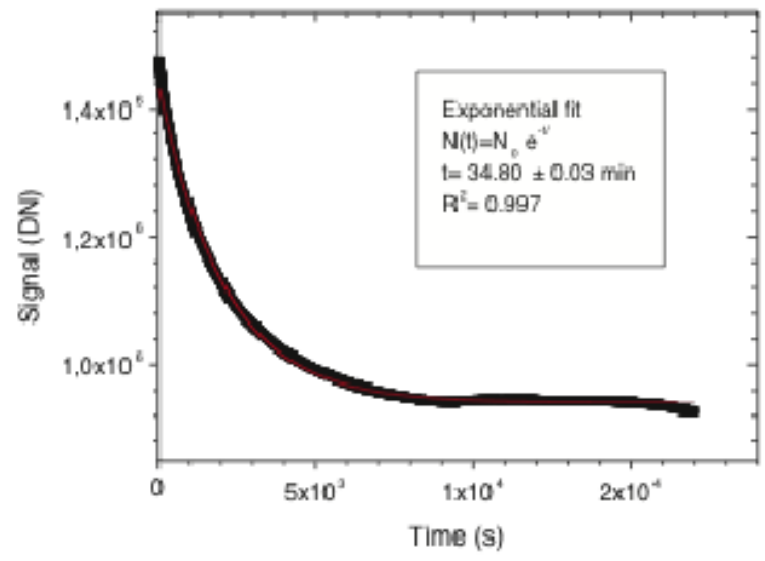

Fig. 3. Decay curve of the chemiluminescent emission:signal generated in the whole imaging array versus elapsed time, measured every $1 \mathrm{~s}$ of exposure after spotting a solution droplet of $70 \mu \mathrm{l}$ of of anti-mouse IgG HRP antibody and activating. The exponential fit is also shown, indicating an experimental life time of $34.80 \pm 0.03 \mathrm{~min}$.

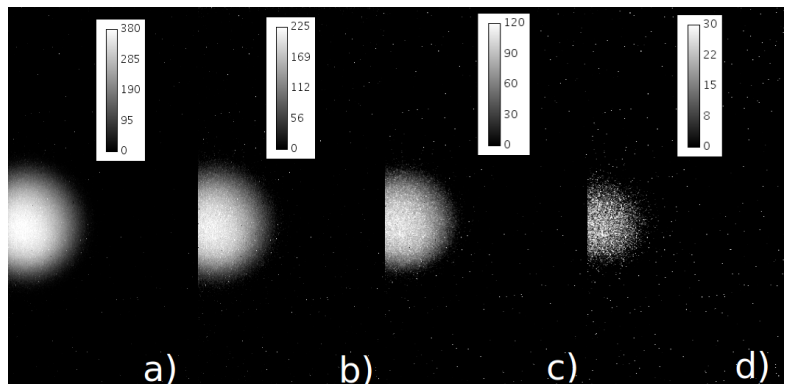

Fig. 4. A drop of anti-mouse IgG HRP antibody actived with Lumi-Light western blotting substrate exposed to the Vanilla sensor for $1 \mathrm{~s}$ for a inferred residual sample mass of $95 \%$ (a), $60 \%$ (b), $37 \%$ (c) $13 \%$ (d). Grey scale represents signal generated in each pixel expressed as Digital Number (DN).

emitted photons in the detector after thresholding according to equation 2 is reported are plotted versus time in fig. 3 . Analysis of the data indicates an exponential decay law for the chemiluminescence process with a life time of $34.80 \pm 0.03$ $\min$.

\section{B. ECL imaging}

In figure 4 the authors show the first chemiluminescence images obtained with a CMOS sensor at room temperature with an exposure time of $1 \mathrm{~s}$ after thresholding. Insets a-d show respectively the chemiluminescent sample after 0.05 , $0.5,1$ and 2 fraction of lifetime, corresponding respectively to a residual antibody mass of $95 \%, 60 \%, 37 \%$ and $13 \%$. The residual antibody mass was inferred by means of the elapsed time from sample activation with Lumi-Light, the life time estimated previously and the initial mass. The grey scale represents the signal in arbitrary Digital Number (DN) generated in each pixel after thresholding according to equation 2.

\section{Linearity, sensitivity and SNR}

The linearity and the sensitivity of the Vanilla sensor has been evaluated for enhanced chemiluminescence, analyzing 


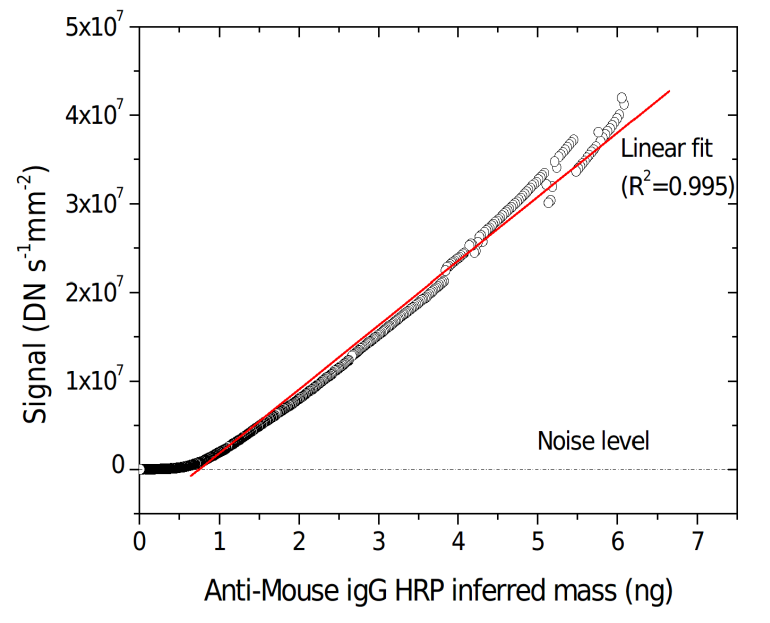

Fig. 5. The relative signal in the ROI after thresholding divided by the exposure time and the area are plotted versus the anti-mouse IgG HRP inferred antibody mass, inferred from the initial mass after exponential decay. A linear fit is shown with a straight line in the linear region. The noise level is also indicated with a dotted line.

the detector response over 5 hours time and calculating the residual anti-mouse IgG HRP antibody inferred mass known the initial mass and the estimated lifetime. Figure 5 shows the detected signal per time unit and area unit in the relevant Region of Interest (ROI) plotted against the residual antimouse IgG HRP antibody inferred mass. Starting from a high mass level the sensitivity curve shows a linear behavior, then approaches a saturation level defined by the noise level. From the data in figures 4, the linearity of the system can be deduced down to a lower limit (Minimum Detectable Activity) of $0.5 \mathrm{ng}$, whereas the higher detection limit was limited by the amount of antibody used $(6.4 \mathrm{ng})$. The antibody mass at which the specific signal rate-versus-activity concentration curve plateau, that is, the maximum value of the linear portion of the sensitivity plot, could not be determined due to the limited activity range of the sample used. The noise level, shown in figure 4 with a dotted line, has been measured independently as $22153 \mathrm{DN} \mathrm{s}^{-1} \mathrm{~mm}^{-2}$. The slope of the linear region of the curve in figure 5 represents the system sensitivity evaluated as $(723.70 \pm 3.14) \times 10^{4} \mathrm{DN} \mathrm{s}^{-1} \mathrm{ng}^{-1} \mathrm{~mm}^{-2}$. Figure 6 shown the Signal-to-Noise Ratio (SNR) as a function of the exposure time (in the range 3-120 s) measured at different fractions of lifetime of the chemiluminescent sample (from 0.05 to 5 lifetime), corresponding to a residual sample mass reported as a percentage in figure. It has been observed that the SNR, at each fixed exposure time, decreases exponentially in proportion to residual mass decreases.

\section{Comparison with film-based images}

A comparative analysis between western blotting images acquired with the Vanilla CMOS APS and conventional film based images has been undertaken in order to evaluate and quantify the improvement in the detection process.

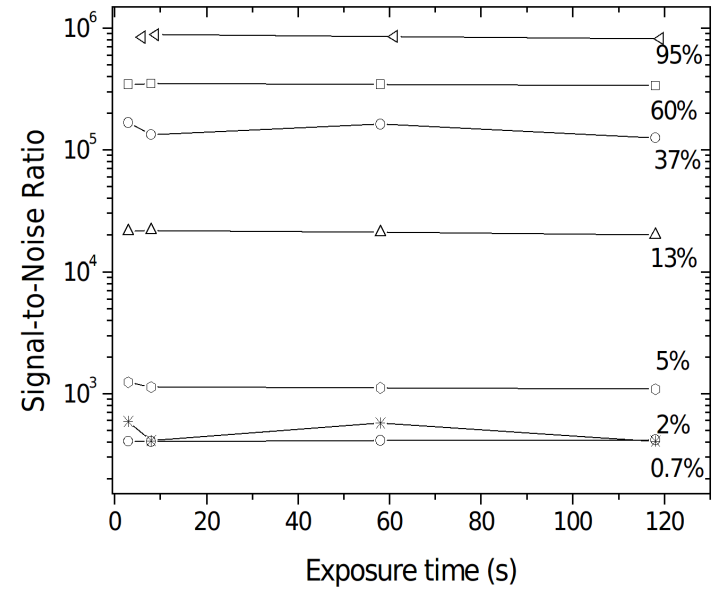

Fig. 6. Signal-to-Noise Ratio versus exposure time measured at several fractions of lifetime corresponding to a residual sample mass reported as percentage in figure.

A set of 32 blots of anti-human IgG HRP antibody ranging from $1.17 \mathrm{ng}$ to $0.39 \mu \mathrm{g}$ was exposed to the Vanilla detector after activation with Lumi-Light. This sample was imaged with conventional film emulsion for $5 \mathrm{~s}$ (see fig. $7(a)$ ) and 1 min (see fig. $7(b)$ ) respectively. Selected blots of this samples were also imaged with the Vanilla sensor in order to compare the response. Blot $I 2$ (2.73 ng of anti-human IgG HRP antibody) and $N 1(2.73 \mu \mathrm{g}$ of anti-human IgG HRP antibody) were imaged with the Vanilla system for the same exposure time used in film images (see fig. $7(c)$ and $(d)$ ) Blot $I 2$ is not visible with film emulsion exposed for $5 \mathrm{~s}$ (fig. $7(a)$ ) whereas it is visible when the blot is imaged with the Vanilla detector with the same exposure time fig. $(7(c))$. The Vanilla sensor showed a higher sensitivity than the conventional film emulsion. Exposing film emulsion for a longer time $(1 \mathrm{~min}$ in fig. $7(b)$ ) allows detection of all the activity values blotted on the sample. Nevertheless saturation occurs. In fact blot $N 1$ is saturated in the film image exposed for $1 \mathrm{~min}$ (fig. $7(b)$ ). The same blot is not saturated when exposed to the Vanilla sensor for the same exposure time (fig. $7(d)$ ) impling a larger dynamic range. Furthermore the two blots under investigation were imaged for $1 \mathrm{~s}$ with the Vanilla system (see fig. $7(e)$ and $(f)$ ) demonstrating that the system is capable to clearly detect both above the noise level.

\section{CONCLUSIONS AND FURTHER WORK}

The first images of ECL detection with a room temperature CMOS detector have been presented and the initial performance of the system have been assessed. Parameters such as sensitivity, linearity, Minimum Detectable Activity (MDA) and Signal-to-Noise ratio (SNR) have been evaluated with antimouse and anti-human IgG HPR antibody labeled with chemiluminescent reagents. The response of the Vanilla detector to chemilunescence light has been compared with conventional film emulsion images. The CMOS APS demonstrated a higher sensitivity and a larger dynamic range compared to film images. 

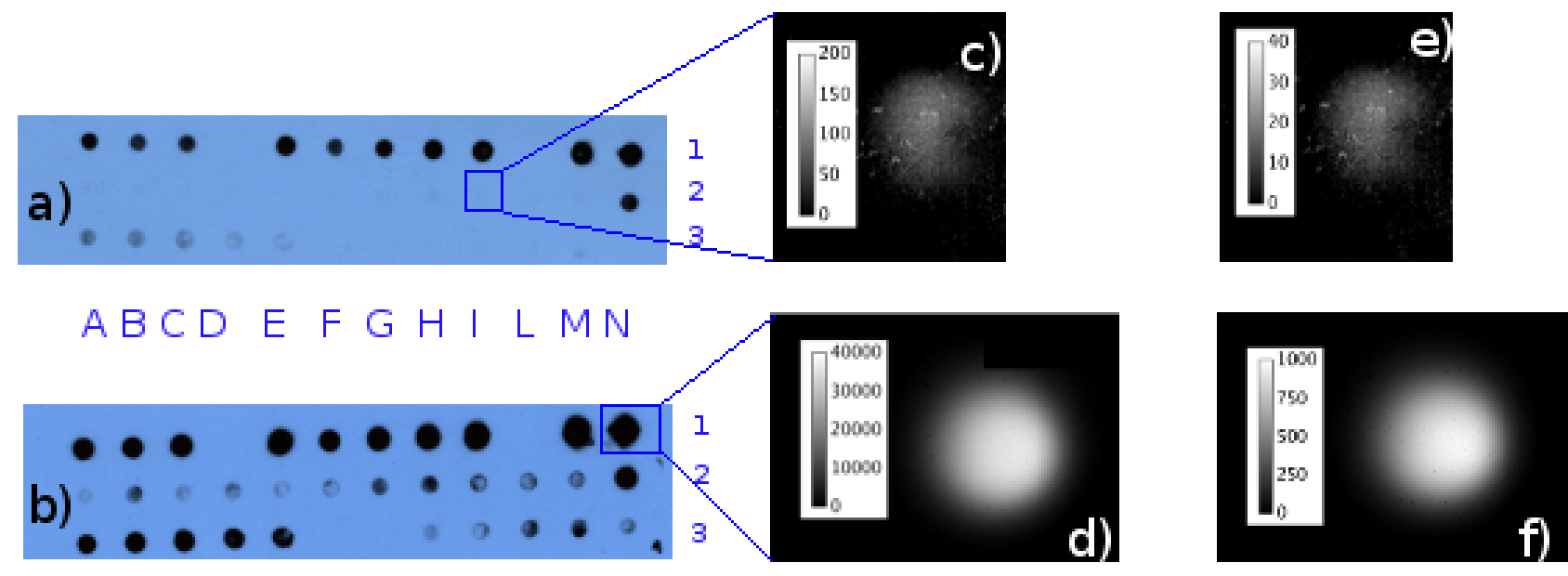

Fig. 7. Film emulsion images of 32 blots of anti-human IgG HRP antibody ranging from $1.17 \mathrm{ng}$ to $0.39 \mu \mathrm{g}$ after activation with Lumi-Light. Samples were exposed to the film emulsion for $5 \mathrm{~s}(a)$ and $1 \mathrm{~min}(b)$ respectively. Blot $I 2(2.73 \mathrm{ng}$ of anti-human IgG HRP antibody) and $N 1$ (2.73 $\mu \mathrm{g}$ of anti-human IgG HRP antibody) were also imaged with the Vanilla detector for $5 \mathrm{~s}(c)$ and $1 \mathrm{~min}(d)$ and $1 \mathrm{~s}(e$ and $f)$ respectively. Grey scale represents signal generated in each pixel expressed as Digital Number (DN).

\section{REFERENCES}

[1] G.H.G. Thorpe and L.J. Kricka, "Enhanced chemiluminescent reactions catalyzed by horseradish peroxidase“, Meth. Enzymol., vol.133, p.331$354,1986$.

[2] A.R. Mahon et al., "A CCD-based system for the detection of DNA in electrophoresis gels by UV absorption", Phys. Med. Biol., vol.44, pp. 1529-41, 1999.

[3] D.A. McGregor and E.S. Yeung, "The interactive control of pulsed field gel electrophoresis via real time monitoring", Anal. Chem., vol.64 pp.16,1992 .

[4] $\operatorname{Ettan}^{T M}$ DIGE, GE Healthcare, 2011, [Online] http://gelifesciences.com.

[5] Chemidoc ${ }^{T M}$ XRS + System, BioRad Laboratories, 2011 [Online], http://bio-rad.com
[6] N.T. Pereira et al., "Chemiluminescent detection of horseradish peroxidase using an integrated amorphous silicon thin-film photosensor", IEEE Sens.Journ., vol.9,No.10, pp.1282-1290,2009.

[7] M. Gersbach et al., "A room temperature CMOS single photon sensor for chemiluminescence detection", presented at International Conference on Miniaturized Systems for Chemistry and Life Science (MicroTas), November 2006.

[8] N. Allinson et al., "The Multidimensional Integrated Intelligent Project (MI-3)“, Nucl. Instr. Meth. A, vol.604 pp.196-198,2009.

[9] J. Cabello et al., " Digital Autoradiography Using Room Temperature CCD and CMOS Imaging Technology“, Phys.Med.Biol., vol. 52(16) pp. 4993-5011,2007. 